\title{
MODELLING AND ESTIMATION OF LEAKAGE PHENOMENA IN MAGNETIC CIRCUITS
}

\author{
ABRAHAM DAUHAJRE AND R.D. MIDDLEBROOK
}

\author{
Power Electronics Group \\ California Institute of Technology
}

\begin{abstract}
A new modelling method for magnetic circuits is presented. The method can be used to model magnetic circuits with any number of windings. The models incorporate adequate information about the correct distribution of leakage energy, the presence of gaps throughout the core, the arrangement of the windings, and the type of core used. Several commonly used arrangements such as toroids with uniformly distributed turns, and bobbin core structures with multiple windings were modelled with the new technique. The measured electric circuit model values always compared favorably with the predicted physical values derived with the new method.
\end{abstract}

\section{$1 \quad$ Introduction}

In recent years, the use of magnetic components in switching converters has been greatly affected by new developments in the disciplines that form the basis for power electronics.

In the field of semiconductor devices, the development of new high-power switching devices (FET's, etc.) prompted designers of power conversion equipment to increase the switching frequency at which the main semiconductor devices are operated. In consequence, the size and weight of the magnetics, and therefore of the entire power supply, have been considerably reduced.

As the switching frequency is increased, a point is reached at which the bottleneck of such designs is not the semiconductor devices but, on the contrary, in the magnetics design.

This work was supported in part by grants from the General Telephone Company, and Emerson Electric Company, by a contract from IBM Owego, and by Caltech's Program in Advanced

Technologies, sponsored by Aerojet General, General Motors, GTE, and TRW.
For example, even after careful layout of the isolation transformer in a switching power supply, a small residual leakage inductance can have severe adverse effects on the power supply operation and design, from requiring sizable power dissipative networks to making the design completely inoperable when the switching frequency is sufficiently increased.

The development of new topologies for switching power converters, together with the discovery of the zero-ripple phenomena in coupled inductors and integrated magnetic structures $[1,2,3]$, have also played a very important role in the development of new magnetic circuits for switching power converters.

For example, in many multiple-output switching converters, the output and input inductors can be combined into a single magnetic structure [4]. The resultant savings in the cost and reduced size of the converters are further augmented by improved performance, through reduced ripple currents. However, the actual ripple current distribution among the various output windings is, to first-order, dependent on the distribution of leakage energy in the windings of the magnetic structure.

Because leakage can have both positive and negative effects in different applications, it is important to know, for each case, what is the correct distribution of leakage energy in the windings of the magnetic structure under investigation.

Conventional modelling methods for magnetic circuits [5] have always fallen short of predicting the correct distribution of leakage energy in the windings of a magnetic structure.

A new modelling method for magnetic circuits is introduced in this paper. The technique involves determination of the approximate flux pattern inside the actual magnetic structure under investigation, and the analytic determination of all the parameter values in the electric circuit model derived from the flux pattern.

This method can be used to model magnetic circuits with any number of windings. The models contain adequate information about the correct distribution of leakage energy, the arrangement of the windings, the presence of gaps throughout the core, and the type of core used. 
The electric circuit models (physical models) obtained with this method are physically natural, i.e., the elements in these models have a one-to-one relationship with corresponding physical quantities in the original magnetic structure.

These models can be particularly useful in prediction of the effects of second-order parasitics on switching converters. For example, for a particular application, different arrangements can be studied through their physical models, and the one that gives the best performance can easily be chosen. All this can be accomplished without going through the usual "trial and error" process.

2

Magnetic Components and Leakage Phenomena in Switching Converters

The effects of magnetic leakage in different types of magnetic components are reviewed in this section. In single-winding inductor applications, the only effect that leakage has (from the circuit point of view) is to increase the effective value of inductance. Other than that it is of little importance. The effects of leakage are far more important in multiple-winding components such as transformers, coupled inductors, and integrated magnetic structures.

The isolated flyback dc-to-dc converter in Fig. 1 uses a two-winding energy storage transformer. The ideal voltage and current waveforms of the transistor switch are also illustrated in Fig. 1. However, owing to the non-zero switching time of the transistor, and the "parasitic" leakage inductance of the transformer, the actual non-ideal current and voltage waveforms of the transistor switch are approximately as shown in Fig. 2. The spurious voltage spike across the transistor collectoremitter junction is approximately given by

$$
\mathrm{v}_{\mathbf{s}} \approx \ell \frac{\mathrm{I}}{\Delta \mathrm{t}}
$$

where $l$ is the effective leakage inductance of the transformer reflected to the primary, I is the current in the primary at turn-off, and $\Delta t$ is the approximate turn-off time of the transistor. Typically, protection circuits are used to prevent a breakdown of the switch owing to the voltage spike.

This is an example of an adverse leakage effect in magnetic components. There are other applications where, to the contrary, leakage is used to improve the performance of the converter. Such is the case of the Cuk converter in Fig. 3, where the input and output inductors are coupled into a single magnetic component. The operation of this converter has been extensively discussed in the literature $[1,2,3]$, where it is shown that proper selection of the leakage parameters in the coupled inductors can lead to zero ac current ripple on either the input or the output of the converter.

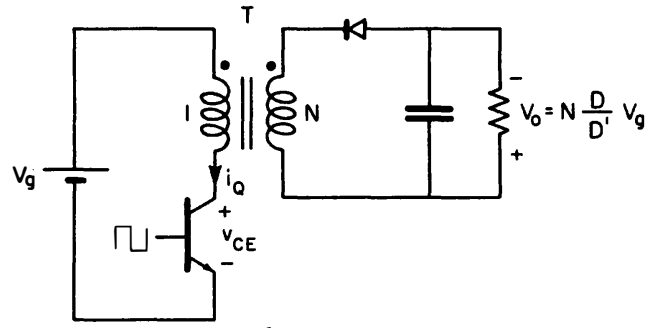

flybock converter

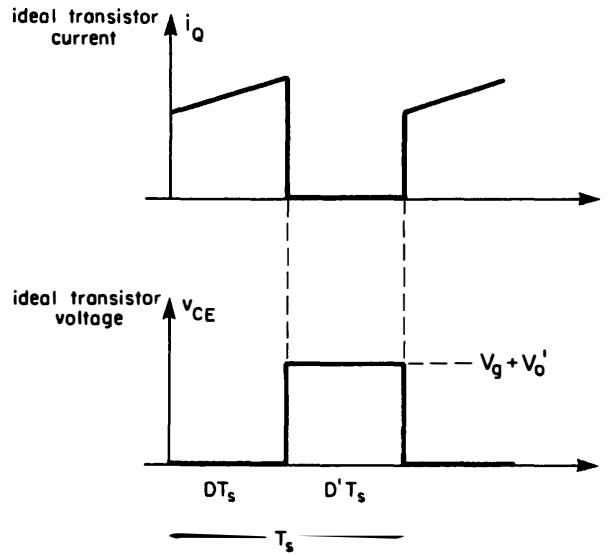

Fig. 1. Illustration of ideal transistor voltage and current waveforms in flyback converter.
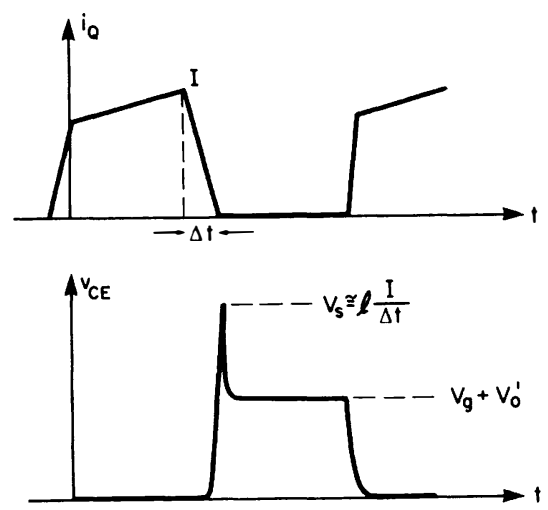

Fig. 2. The non-ideal voltage and current waveforms of the transistor illustrate the adverse effects of the leakage inductance on the switching element. 


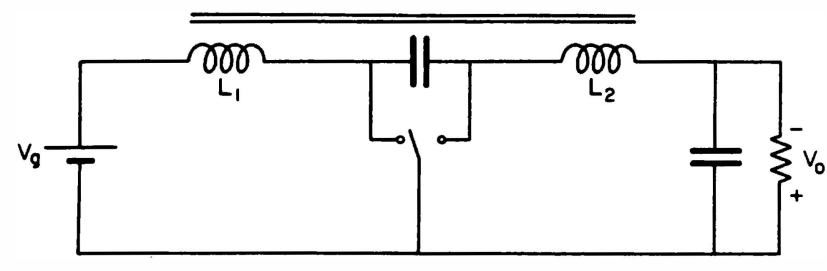

Fig. 3. Cuk dc-to-dc converter with coupled inductors.

In isolation transformers and coupled inductors with multiple windings, such as those in the forward converter in Fig. 4, it is not so well understood how the different leakage parameters affect the performance of the converter. The main reason for this is the lack of "proper" electric circuit models for multiple-winding structures.

Proper electric circuit models for multiple winding magnetic structures that incorporate adequate information about the correct distribution of leakage energy in the windings can be very helpful in the design of transformers for minimum leakage effects, as well as coupled inductors for minimum ac ripple currents.

Solving the leakage problem in general involves determination of proper electric circuit models where the different inductance parameters (including those associated with magnetic leakage) are analytically estimated in terms of the geometry of the magnetic structure. A simple method for estimating the approximate leakage energy in the windings of a magnetic structure is reviewed in the next section.

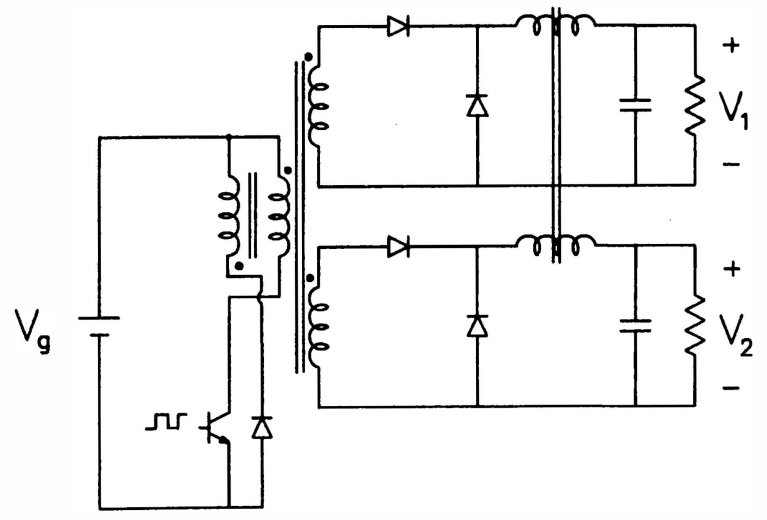

Fig. 4. Dual-output isolated forward converter with output inductors coupled.

\section{$3 \quad$ Energy in Magnetic Circuits}

The magnetic energy in a volume of material $\mathrm{v}$ where the magnetic field intensity $\mathrm{H}$ is known is given by

$$
E=\frac{\mu}{2} \iiint_{V} H^{2} \mathrm{dv}
$$

If the volume $v$ corresponds to the winding of a magnetic circuit which carries a current $I$, the magnetic energy in Eq. (2) can also be expressed as

$$
E=\frac{1}{2} l I^{2}
$$

where $l$ is the "effective" inductance of the winding. Comparison of Eqs. (2) and (3) gives

$$
\ell=\frac{\mu_{\mathrm{o}}}{\mathrm{I}^{2}} \iiint_{\mathrm{v}} \mathrm{H}^{2} \mathrm{dv}
$$

where the integral is evaluated throughout the volume of the winding $\mathrm{v}$.

In general, Eq. (4) can be used to compute the "effective" or "equivalent" inductance of any part of a magnetic structure for which the field intensity $\mathrm{H}$ has previously been determined. This is illustrated next with a simple example.

\subsection{Toroid with Uniformly Distributed Turns}

To illustrate the use of Eq. (4), a simple toroidal core with $\mathrm{N}$ uniformly distributed turns is used as an example, and is shown in Fig. 5.

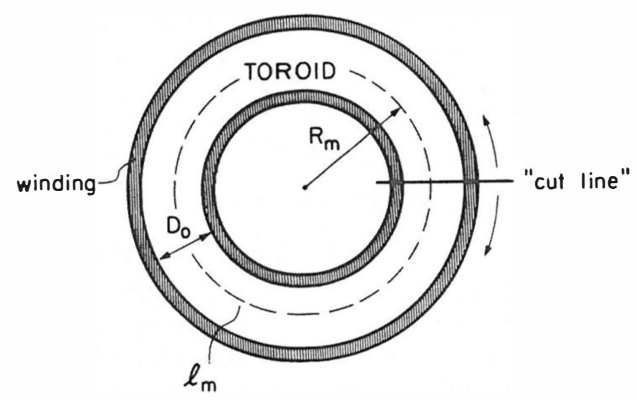

Fig. 5. Toroid with uniformly distributed turns.

The field intensity inside the toroid $\mathrm{H}_{\mathrm{i}}$ can be obtained using Ampere's law in its most general form

$$
\oint_{C} \mathrm{H} \cdot \mathrm{d} \ell=\iint \mathrm{Jd} s
$$


which basically states that the closed line integral of $\mathrm{H}$ is equal to the total current enclosed by the integration path $c$. For the example in Fig. 5, the integration path $c$ goes along the center of the toroid. The total current enclosed by $c$ is therefore

$$
\iint \mathrm{J} \cdot \mathrm{ds}=\mathrm{NI}
$$

If the toroid is made of ferromagnetic material, the field intensity $\mathrm{H}_{\mathrm{i}}$ can be assumed "almost" uniform throughout the toroid, and parallel to the path $c$. Under these conditions, Eqs. (5) and (6) give

$$
\mathrm{H}_{\mathrm{i}}=\frac{\mathrm{NI}}{\ell_{\mathrm{m}}}
$$

where $\ell_{m}=2 \pi R_{m}$ is the mean length of the magnetic path of the toroid.

Equation (7) gives the approximate field intensity inside the toroid. To estimate the field intensity across the winding, it is assumed that the turns are made of thin wires, and therefore the shape of the winding can be assumed to be that of a thick uniform layer around the toroid which carries a total current NI. To visualize the next steps more easily, the toroid is "cut" and stretched open as shown in Fig. 6(a). The winding is now a cylindrical conductor of thickness $h$ and of internal radius $r_{0}$, which surrounds the toroid.

The field intensity $\mathrm{H}(\rho)$ inside the winding (leakage field intensity) can be obtained using Ampere's law (Eq. (5)), where the closed integration path $c$ can be selected as shown in Fig. 6(a). The selected path is closed because the bottom of the figure is actually connected to the top.

If $\mathrm{H}(\rho)$ is assumed normal to the current density J, the left side of Eq. (5) becomes

$$
\oint_{C} \mathrm{H} \cdot \mathrm{d} \ell=\int_{\ell=0}^{\ell=\ell_{\mathrm{m}}} \mathrm{H}(\rho) \mathrm{d} \ell=\mathrm{H}(\rho) \ell_{\mathrm{m}}
$$

The right hand side of Eq. (5) is the total current enclosed by the integration path $c$. If the current density $J$ is constant throughout the entire cross section of the conductor cylinder, the total current enclosed by $c$ is then

$$
I_{c}=J(h-\rho) l_{m}
$$

where

$$
\mathrm{J}=\frac{\mathrm{NI}}{\mathrm{h} \ell_{\mathrm{m}}}
$$

and $\mathrm{h} \ell_{\mathrm{m}}$ is the area of the cross section of the conductor cylinder. Substitution of Eq. (10) into (9) gives

$$
I_{c}=N I\left(1-\frac{\rho}{h}\right)
$$

(a)

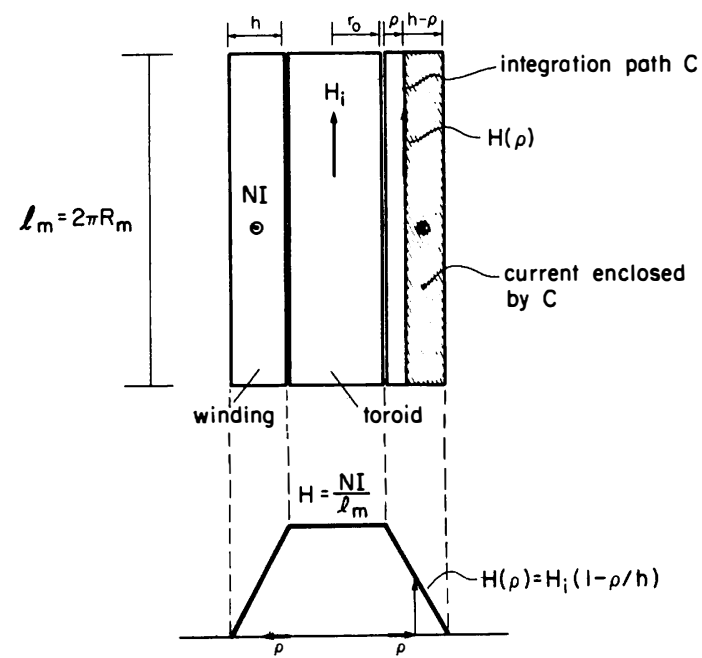

Fig. 6. "Cut" and stretched toroid (a) and field intensity across the winding and the toroid (b).

Finally, from Eqs. (8) and (11)

$$
\mathrm{H}(\rho)=\mathrm{H}_{\mathrm{i}}\left(1-\frac{\rho}{\mathrm{h}}\right)
$$

where $\mathrm{H}_{\mathrm{i}}=\mathrm{NI} / \ell_{\mathrm{m}}$ is the field intensity inside the toroid.

Figure 6(b) illustrates the variation of the field intensity $\mathrm{H}$ inside and outside the toroid. At the boundary between the toroid and the winding $(\rho=0)$, the field intensity is $H_{i}$. This is as expected since one of the boundary condition for magnetic fields is that tangential components of the field intensity are equal on both sides of a boundary. Finally, at the end of the winding $(\rho=\mathrm{h})$, the field intensity reduces to zero.

Finally, if the toroid is closed back into its original circular form, it is possible to draw the direction of the fluxes inside the winding and the toroid. This is shown in Fig. 7.

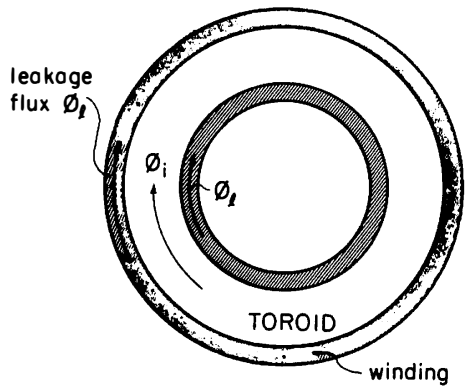

Fig. 7. The toroid and the winding in Fig. 6 can be closed back into their original circular form to illustrate the direction of the flux inside the toroid $\phi_{i}$, and the leakage flux $\phi_{\ell}$. 


\section{Inductance Calculation}

Equation (4) can now be used to find both the inductance of the toroid as well as the "equivalent" or "effective" inductance of the winding, i.e., the leakage inductance. The inductance of the toroid is found by substitution of Eq. (7) into (4) and evaluation of the integral throughout the volume of the toroid. This gives

$$
\mathrm{L}=\frac{\mathrm{N}^{2}}{R_{\mathrm{m}}}
$$

where $R_{\mathrm{m}}=\mu \mathrm{S} / \ell_{\mathrm{m}}$ is the reluctance of the toroid.

The effective (leakage) inductance of the winding is computed by substitution of $\mathrm{H}(\rho)$ from Eq. (12) into Eq. (4), and evaluation of the integral throughout the volume of the winding, which gives

$$
\ell=\frac{2}{3} \mathrm{~N}^{2} \pi \mu_{\circ} \frac{\mathrm{h}}{\ell_{\mathrm{m}}}\left(\mathrm{r}_{\mathrm{o}}+\frac{\mathrm{h}}{4}\right)
$$

where $l$ is the effective leakage inductance of the winding. Finally, the total inductance of the structure is the sum of the inductance of the toroid L (Eq. (13)), and the leakage inductance $\ell$ (Eq. (14)).

\subsection{Multiple-Winding Structures and the General Solution to the Leakage Problem}

The previous inductor example illustrates how to obtain simple analytic approximations for the field intensity inside the windings of a magnetic circuit. It also shows how Eq. (4) can be used to compute the effective inductance of some volume of material for which the field intensity has previously been determined. However, for multiple-winding structures it is necessary not only to estimate the energy of the magnetic fields inside the windings, but also to know how this energy is distributed among the different windings.

The general solution to the leakage problem is divided into various steps. These steps involve first the determination of a "physical" electric circuit model, and finally the estimation of the parameters in the model (including all leakage inductances).

In the past magnetic circuits have been modelled based on mathematical descriptions of "generic" magnetic circuits with the same number of windings [5]. Unfortunately, these mathematical models have always fallen short of predicting the correct distribution of leakage energy in the windings of a magnetic structure. The next section reviews the concepts and steps involved in determination of these mathematical models.

\section{$4 \quad$ Mathematical Models for Magnetic Circuits}

The conventional method for modelling magnetic circuits [5] is reviewed in this section. It is shown with some examples that although these models are conceptually right, the meaning of the models and its elements can sometimes be reduced to mere mathematical abstractions with no physical interpretation.

\subsection{Mathematical Description of Magnetic Circuits}

Figure 8 shows a simple two-winding transformer. One possible way to model such a structure is to consider the two-winding toroid shown in Fig. 9 a "generic" case for all twowinding transformers. Figure 9 also shows the assumed flux pattern inside the generic toroid and its windings.

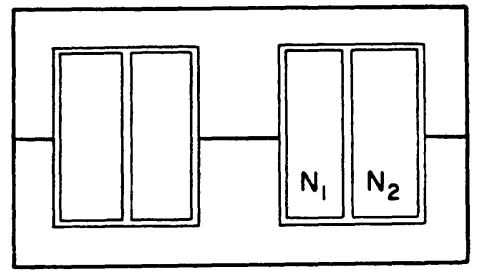

Fig. 8. Two-winding side by side bobbin core arrangement.

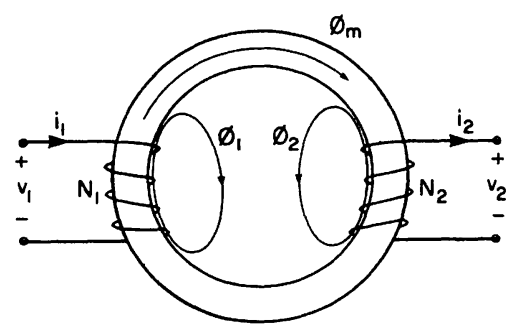

Fig. 9. Generic two-winding transformer.

The total flux enclosed by $\mathrm{N}_{1}$ is

$$
\phi_{\mathrm{N} 1}=\phi_{\mathrm{m}}+\phi_{1}
$$

Similarly the total flux enclosed by $N_{2}$ is

$$
\phi_{\mathrm{N} 2}=\phi_{\mathrm{m}}-\phi_{2}
$$

Using Faraday's law one can also write

$$
\begin{aligned}
& \mathrm{v}_{1}=\mathrm{N}_{1} \frac{\mathrm{d} \phi_{\mathrm{N} 1}}{\mathrm{dt}}=\mathrm{N}_{1}\left(\frac{\mathrm{d} \phi_{\mathrm{m}}}{\mathrm{dt}}+\frac{\mathrm{d} \phi_{1}}{\mathrm{dt}}\right) \\
& \mathrm{v}_{2}=\mathrm{N}_{2} \frac{\mathrm{d} \phi_{\mathrm{N} 2}}{\mathrm{dt}}=\mathrm{N}_{2}\left(\frac{\mathrm{d} \phi_{\mathrm{m}}}{\mathrm{dt}}-\frac{\mathrm{d} \phi_{2}}{\mathrm{dt}}\right)
\end{aligned}
$$


The leakage fluxes $\phi_{1}$ and $\phi_{2}$ can also be expressed as

$$
\begin{aligned}
& \phi_{1}=N_{1} \rho_{1} i_{1} \\
& \phi_{2}=N_{2} \rho_{2} i_{2}
\end{aligned}
$$

where $\rho_{1}$ and $\rho_{2}$ are the permeances of the magnetic paths of $\phi_{1}$ and $\phi_{2}$ [5].

By Lenz's law the relation between $\phi_{\mathrm{m}}$ and the currents $i_{1}$ and $i_{2}$ can be written as

$$
\phi_{\mathrm{m}}=\rho_{\mathrm{m}}\left(\mathrm{N}_{1} i_{1}-\mathrm{N}_{2} i_{2}\right)
$$

where $\beta_{m}$ is the permeance of the path of the magnetization flux $\phi_{\mathrm{m}}$ [5]. Substitution of Eqs. (19), (20), and (21) into Eqs. (17) and (18) and rearrangement of similar terms gives

$$
\begin{aligned}
& v_{1}=N_{1}^{2} \rho_{1} \frac{d i_{1}}{d t}+N_{1}^{2} \rho_{m}\left(\frac{d i_{1}}{d t}-\frac{N_{2}}{N_{1}} \frac{d i_{2}}{d t}\right) \\
& v_{2}=-N_{2}^{2} \rho_{2} \frac{d i_{2}}{d t}+N_{2} N_{1} \rho_{m}\left(\frac{d i_{1}}{d t}-\frac{N_{2}}{N_{1}} \frac{d i_{2}}{d t}\right)
\end{aligned}
$$

The unit for permeance $\beta$ is Henry/turns ${ }^{2}$, and consequently the following inductances can be defined in terms of the permeances $P_{m}, P_{1}$, and $P_{2}$ :

$$
\begin{aligned}
& \mathrm{L}_{\mathrm{m}}=\mathrm{N}_{1}{ }^{2} \mathrm{p}_{\mathrm{m}} \\
& \ell_{1}=\mathrm{N}_{1}{ }^{2} \mathrm{P}_{1} \\
& \ell_{2}=\mathrm{N}_{2}{ }^{2} \mathrm{P}_{2}
\end{aligned}
$$

Substitution of Eqs. (24), (25), and (26) into Eqs. (22) and (23) gives

$$
\begin{aligned}
& \mathrm{v}_{1}=\ell_{1} \frac{\mathrm{di}_{1}}{\mathrm{dt}}+\mathrm{L}_{\mathrm{m}}\left(\frac{\mathrm{di_{1 }}}{\mathrm{dt}}-\frac{\mathrm{N}_{2}}{\mathrm{~N}_{1}} \frac{\mathrm{di}_{2}}{\mathrm{dt}}\right) \\
& \mathrm{v}_{2}=-l_{2} \frac{\mathrm{di}_{2}}{\mathrm{dt}}+\frac{\mathrm{N}_{2}}{\mathrm{~N}_{1}} \mathrm{~L}_{\mathrm{m}}\left(\frac{\mathrm{di} \mathrm{i}_{1}}{\mathrm{dt}}-\frac{\mathrm{N}_{2}}{\mathrm{~N}_{1}} \frac{\mathrm{di}}{\mathrm{dt}}\right)
\end{aligned}
$$

Equations (27) and (28) constitute a mathematical description of the input-output characteristics of the generic transformer in Fig. 9 .

\section{Electric Circuit Model}

An equivalent electric circuit model that satisfies Eqs. (27) and (28) is illustrated in Fig. 10. This model is known as the $\pi$-model for a two-winding transformer.

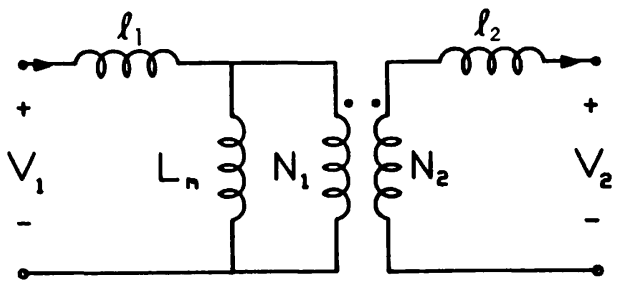

Fig. 10. Electric circuit model ( $\pi$-model) for generic two-winding transformer in Fig. 9 .
The $\pi$-model in Fig. 10 can be mathematically forced to satisfy the inputoutput characteristics of the two-winding transformer in Fig. 8. However, since the elements in this model represent physical quantities associated with the generic transformer in Fig. 9 and not the actual one in Fig. 8, the meanings of the elements in this model are reduced to mere mathematical abstractions with no physical interpretation. In general, for any two-winding transformer configuration other than the generic two-winding transformer in Fig. 9, the $\pi$-model is just a mathematical description i.e., a mathematical model of the transformer characteristics.

\subsection{Mathematical Models for Three-Winding Magnetic Circuits}

The same procedure used to model the generic two-winding transformer can be used to find an electric circuit model for the generic three-winding transformer shown in Fig. 11.

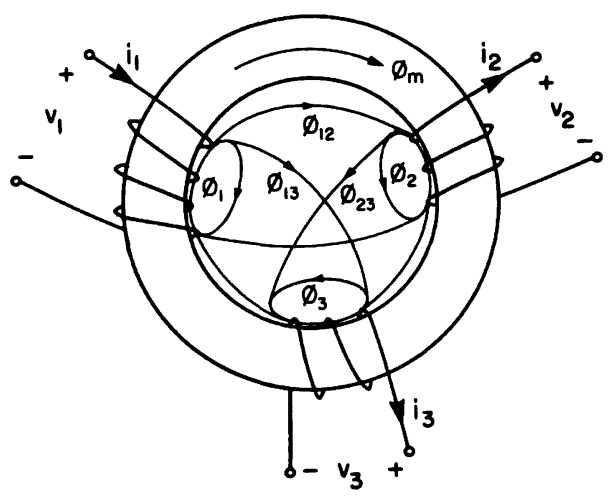

Fig. 11. Generic three-winding transformer with the seven flux components illustrated.

Because of the additional winding $\mathrm{N}_{3}$ there are several additional flux components. The seven flux components are illustrated in Fig. 11. The electric circuit model, which is derived in a similar way as for the two-winding transformer, is illustrated in Fig. 12. The expressions for the parameters in the model are given in [5] and are mathematical functions of all the permeances of the different flux paths in the generic toroid.

For example, the expression for the "mutual inductance" $M_{0}$ is given by

$$
M_{0}=N_{1}^{2} \frac{\left(P_{m}+P_{12}\right)\left(P_{m}+P_{13}\right)}{\left(P_{m}+P_{23}\right)}
$$




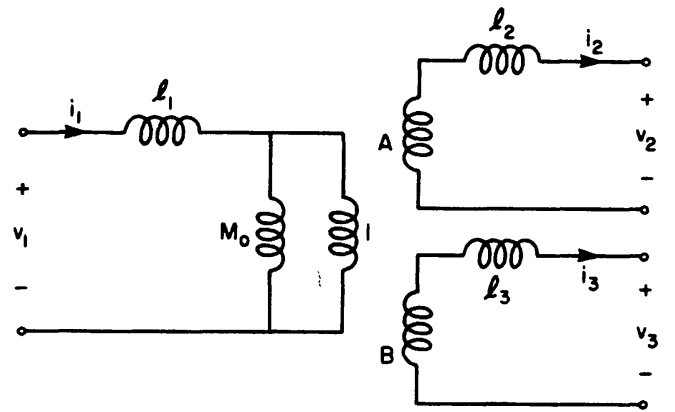

Fig. 12. Electric circuit model for threewinding generic transformer in Fig. 11 .

Clearly, the complicated nature of the expressions for the parameters in the model makes a general and simple interpretation of the electric circuit model in terms of the generic three-winding transformer not always possible. Furthermore, if this model is used to characterize a "real" three-winding structure such as that in Fig. 13, any interpretation is almost hopeless.

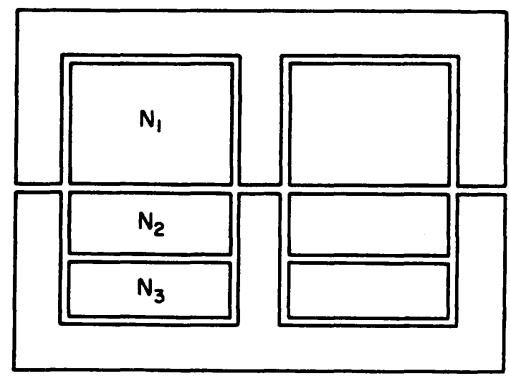

Fig. 13. Three-winding bobbin core arrangement.

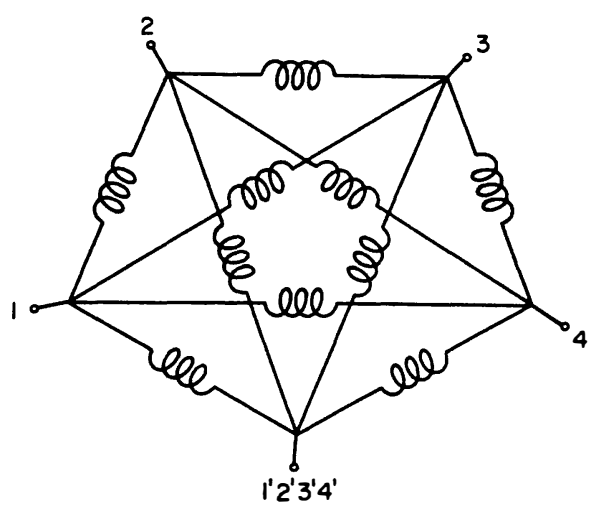

Fig. 14. Electric circuit model (mathematical mode1) for four-winding transformer.

\subsection{Mathematical Models for Four-Winding Magnetic Circuits}

Unfortunately, for any transformer with more than three windings it is not possible to represent in general the input-output characteristics with a $\pi$-model. The general electric circuit model (mathematical model) for a four-winding transformer is obtained in [5] and is shown in Fig. 14. Any general

interpretation from this model is almost hopeless.

With respect to design of the magnetic structure, mathematical models are in general not very useful. They do not contain adequate information about the physical distribution of leakage energy in the windings, about the presence of gaps throughout the core, and in general, about the geometric and structural properties of the magnetic component under investigation.

Electric circuit models which are derived from the actual magnetic structures under investigation, and are such that the parameters in the models (leakage inductances, etc) have a one-to-one relationship with corresponding physical quantities in the original magnetic structure, can be used not only as analysis tools, but also as design oriented tools for magnetic structures.

These models which are physically natural are the main topic of this paper. The general method to obtain the physical model of a magnetic structure is discussed in the next section.

$5 \quad$ Physical Description of Magnetic Circuits: Physical Models

Mathematical models are based on generic magnetic circuits with the same number of windings as the original magnetic structure. Physical models, on the other hand, are based fundamentally on a knowledge of the distribution and intensity of the fluxes inside the actual magnetic structure under investigation. To obtain the physical model of a magnetic structure a new modelling technique is used.

The general modelling procedure for physical models is illustrated in Fig. 15. The first step involves the determination of the flux pattern inside the actual magnetic. structure under investigation. Based on this pattern a set of algebraic flux equations are derived. These equations, which simply describe the distribution of the fluxes inside the structure, are used to construct the reluctance circuit model for the magnetic structure. The electric circuit model is then derived from the reluctance model using the principle of duality $[6,7]$. Finally, all the parameter values in the electric circuit model are analytically determined in terms of the geometry of the structure.

To illustrate the method the two-winding side by side bobbin core arrangement in Fig. 8 is used as an example. What follows is a description of each one of the steps involved in determination of the physical model for the transformer in Fig. 8. 


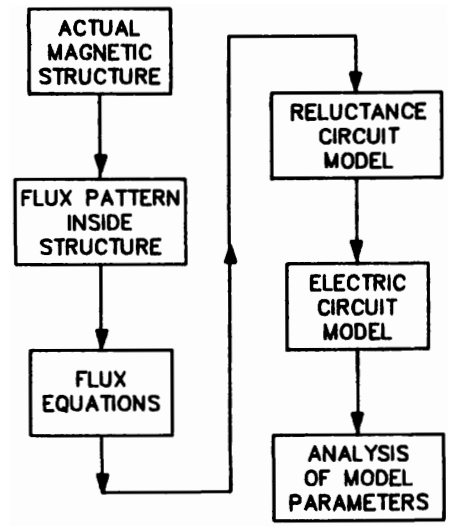

Fig. 15. General procedure to obtain the physical model of a magnetic circuit.

\subsection{Approximate Flux Pattern Inside The Magnetic Structure}

To obtain the physical model of a magnetic structure it is necessary to find approximately the flux pattern everywhere inside the actual structure under investigation. The flux pattern inside a magnetic structure can in general be obtained using Ampere's law in its most general form, i.e. Eq. (5). For the transformer in Fig. 8 the integration path $c$ can be selected as shown in Fig. 16.
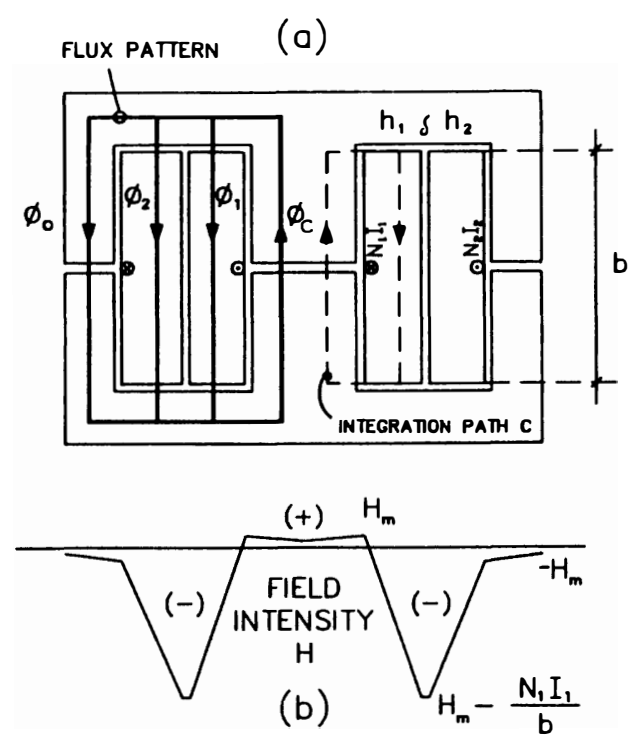

Fig. 16. Illustration of the flux pattern inside two-winding side by side arrangement (a). It is determined with the use of the field intensity plot in (b). Also shown are the infinitely small sources of mmf $N_{1} I_{1}$ and $\mathrm{N}_{2} \mathrm{I}_{2}$.
Use of the integration path shown in Fig. 16, and evaluation of Ampere's law (Eq. (5)) results in

$$
\mathrm{H}(\rho)=\mathrm{H}_{\mathrm{m}}-\frac{\mathrm{N}_{1} \mathrm{I}_{1}}{\mathrm{~b}} \frac{\rho}{\mathrm{h}_{1}}
$$

where $H(\rho)$ is the field intensity inside the primary at a radial distance $\left(\rho+r_{0}\right)$ from the center of the core, and $\mathrm{H}_{\mathrm{m}}$ is the average field intensity inside the core, which is approximately given by

$$
\mathrm{H}_{\mathrm{m}}=\frac{\mathrm{N}_{1} \mathrm{I}_{1}-\mathrm{N}_{2} \mathrm{I}_{2}}{l_{\mathrm{m}}}
$$

The field intensity through the secondary winding and the rest of the core is obtained in a similar way. The complete result is illustrated in Fig. 16(b). The sign of the field intensity determines the direction of the fluxes inside the windings and the rest of the core. This in turn is used to establish the approximate flux pattern inside the structure, which is also illustrated in Fig. 16(a).

\subsection{The Flux Equations}

The next step to determine the physical model of a magnetic circuit is to write down a set of algebraic flux equations which are based on the flux pattern previously determined and on the following definitions.

The total flux enclosed by the primary winding is defined as that which is enclosed by the infinitely small source $N_{1} I_{1}$ shown in Fig. $16(a)$.

$\phi_{\mathrm{N}_{1}} \equiv$ Total flux enclosed by $\mathrm{N}_{1}=\phi_{\mathrm{c}}$

Similarly, the total flux enclosed by the secondary $\mathrm{N}_{2}$ is the sum of all the flux enclosed by the infinitely small source $\mathrm{N}_{2} \mathrm{I}_{2}$ shown in Fig. 16(a).

$$
\phi_{\mathrm{N} 2}=\phi_{\mathrm{c}}-\left(\phi_{1}+\phi_{\delta}+\phi_{2}\right)
$$

In defining these two equations two fundamental approximations are made. The first one incorporates all the $\mathrm{mmf}$ of the windings into the infinitely small sources $\mathrm{N}_{1} \mathrm{I}_{1}$ and $\mathrm{N}_{2} \mathrm{I}_{2}$. The ideal situation would be to consider each turn as a separate source of $\mathrm{mmf}$. This can unnecessarily complicate the results and make the process intractable.

An intermediate solution is to break the windings into their separate layers, as they are physically wound. Each layer would then be treated as a separated winding each carrying a current $\mathrm{N}_{1} \mathrm{I}$, where $\mathrm{N}_{1}$ is the number of turns in the $i^{\text {th }}$ layer, and $I$ is the current of each turn in the layer. The case where the windings are broken into their separate layers is discussed in detail in [7].

The second approximation has to do with the positions of the infinitely small sources $\mathrm{N}_{1} \mathrm{I}_{1}$ and $\mathrm{N}_{2} \mathrm{I}_{2}$ at the extremes of the windings as indicated in Fig. 16(a). The purpose of this is to ensure that all the leakage fluxes $\phi_{1}$ and $\phi_{2}$ are contained within these two sources. This approximation can also be significantly improved by breaking the winding into layers [7]. 
Later in the paper experimental results show that both of these approximations do not introduce significantly large errors in the predicted models. However, if more accurate models are desired, the layer-to-layer models discussed in [7] provide a better answer. Substitution of Eq. (32) into (33) gives

$$
\phi_{\mathrm{N} 2}=\phi_{\mathrm{N} 1}-\phi_{\ell}
$$

where $\phi_{\ell}=\phi_{1}+\phi_{\delta}+\phi_{2}$.

Finally, the last flux equation gives the relation between the flux through the center leg of the core $\phi_{\mathrm{c}}$, and the flux through the outer legs $\phi_{0}$. That is,

$$
\phi_{\circ}=\phi_{c}-\phi_{l}=\phi_{\mathrm{N} 2}
$$

\subsection{Construction of the Reluctance Model}

The reluctance model gives a "circuit like" description of the distribution of the fluxes inside the magnetic structure.

The currents in the reluctance circuit represent the different flux quantities in the magnetic structure which are also described by the flux equations. The mmf sources translate into voltage sources as described in [7]. Each node in the reluctance model satisfies one of the flux equations, and each branch of the reluctance circuit is associated with the corresponding flux path in the magnetic structure. The reluctances represent the the ability of the structure to store magnetic energy along that particular path.

The complete reluctance circuit model for the two-winding side by side arrangement is illustrated in Fig. 17.

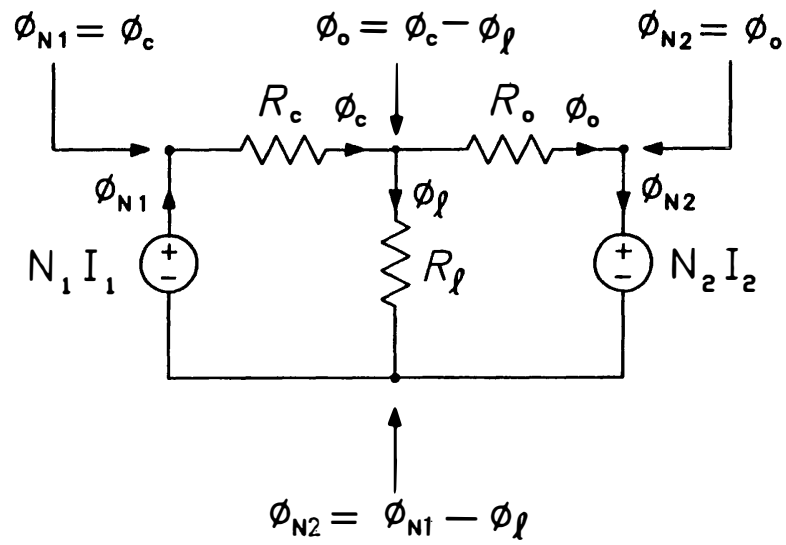

Fig. 17. Reluctance circuit model for twowinding side by side arrangement.
The flux quantities $\phi_{\mathrm{N} 1}$ and $\phi_{\mathrm{N} 2}$, which were previously defined in Eqs. (32) and (33), are the "currents" associated with the "voltage sources" $N_{1} I_{1}$ and $N_{2} I_{2}$, as illustrated in Fig. 17. Notice that the "current" $\phi_{\mathrm{N} 2}$ in the figure together with the sign of the "voltage source" $\mathrm{N}_{2} \mathrm{I}_{2}$ indicate the "load nature" of $\mathrm{N}_{2} \mathrm{I}_{2}$.

The reluctances in the circuit represent the ability of the structure to store energy along that particular path. For instance, $R_{\ell}$ represents the ability of the structure to store energy in the windings, $R_{\mathrm{c}}$ is the reluctance of the center leg gap, and $R_{\circ}$ that of the outer legs.

\subsection{Electric Circuit Model (Physical Mode1)}

In $[6,7]$ a method for obtaining the electric circuit model given the reluctance circuit is reviewed. The technique is known as the principle of duality.

The electric circuit model derived from the reluctance model in Fig. 17 using the principle of duality is illustrated in Fig. 18.

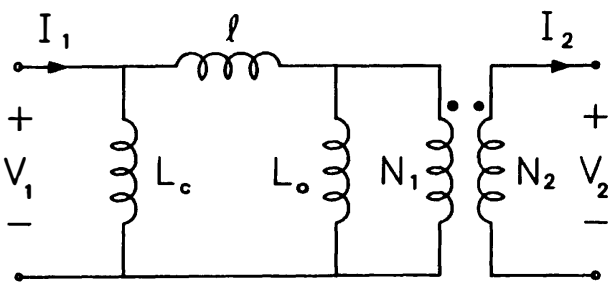

Fig. 18. Electric circuit model for twowinding side by side arrangement.

The relations between the inductances in the electric circuit model and the reluctance elements in the reluctance model are given by

$$
\begin{aligned}
\mathrm{L}_{\mathrm{c}} & =\frac{\mathrm{N}_{1}{ }^{2}}{R_{\mathrm{c}}} \\
\mathrm{L}_{\mathrm{o}} & =\frac{\mathrm{N}_{1}{ }^{2}}{R_{\mathrm{o}}} \\
\ell & =\frac{\mathrm{N}_{1}{ }^{2}}{R_{\ell}}
\end{aligned}
$$

For simplicity, the resistances of the windings are ignored but they can be accounted for in the usual way.

\subsection{Analytic Estimation of the Parameters in the Electric Circuit Mode1}

To completely define the electric circuit model it is necessary to find approximate analytic expressions for all the inductances in the model. 
The two inductances $\mathrm{L}_{c}$ and $\mathrm{L}_{\mathrm{o}}$ in the electric circuit model are easily obtained since they are directly associated with the center and outer leg gaps respectively. That is,

and

$$
\mathrm{L}_{\mathrm{c}}=\frac{\mathrm{N}_{1}^{2}}{R_{\mathrm{c}}}=\frac{\mathrm{N}_{1}^{2}}{\ell_{\mathrm{g}} / \mu_{\mathrm{o}} \mathrm{S}_{\mathrm{c}}}
$$

$$
\mathrm{L}_{\mathrm{o}}=\frac{\mathrm{N}_{1}^{2}}{R_{\mathrm{o}}}=\frac{\mathrm{N}_{1}{ }^{2}}{\ell_{\mathrm{g}} / \mu_{\mathrm{o}} \mathrm{S}_{\mathrm{o}}}
$$

where $S_{c}$ is the effective area of the center leg

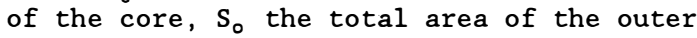
legs, and $\ell_{g}$ the length of the spacer gap.

The leakage inductance $l$ can be calculated by shorting the secondary and exciting the primary with a current source. If the leakage inductance $l$ is much smaller than $\mathrm{L}_{c}$, most of the energy from the current source is stored in the leakage inductance $l$ and not in $\mathrm{L}_{\mathrm{c}}$. The field intensity through the windings can be determined as before. The result is very similar to the plot in Fig. 16(b), except that now the magnetizing current is approximately zero $\left(N_{1} I_{1} \approx N_{2} I_{2}\right)$, and therefore the magnetizing field intensity $H_{m}$ (Eq. (31) is also reduced to approximately zero.

Evaluation of Eq. (4) throughout the volume of the windings under these conditions gives

$$
\begin{aligned}
\ell=2 \pi \mu_{\circ} \mathrm{N}_{1}^{2} \frac{1}{\mathrm{~b}}( & \mathrm{h}_{1}\left(\frac{\mathrm{r}_{\mathrm{o}}}{3}+\frac{\mathrm{h}_{1}}{4}\right)+ \\
& +\delta\left(\mathrm{r}_{\mathrm{o}}+\mathrm{h}_{1}+\frac{\delta}{2}\right)+ \\
& \left.+\mathrm{h}_{2}\left(\frac{\mathrm{r}_{\mathrm{o}}+\mathrm{h}_{1}+\delta}{3}+\frac{\mathrm{h}_{2}}{12}\right)\right)
\end{aligned}
$$

If the widths of the windings are the same, i.e., $h_{1}=h_{2} \neq h / 2$, and $\delta \ll h, E$ (41) reduces to

$$
\ell=\frac{1}{3} \mu_{\circ} \mathrm{N}_{1}{ }^{2} \frac{\mathrm{h}}{\mathrm{b}} \mathrm{MLT}
$$

where MLT $=2 \pi\left(r_{0}+\hbar / 2\right)$ Is the mean length per turn of the bobbin.

The electric circuit model in Fig. 18 together with the expressions given by Eqs . (39), (40), and (41) completely define the physical model for the two-winding side by side arrangement in Fig. 8 .

\subsection{Examples}

Various bobbin cores of different sizes were wound as shown in Fig. 8. Two examples are given in this section.

The first example is a small P-2213 pot core with a spacer gap. The length of the gap and the size of the windings and the core are summarized below.

$\mathrm{N}_{1}=65, \quad \mathrm{~N}_{2}=61$

$r_{0}=$ radius of bobbin $=0.5 \mathrm{~cm}$

$\mathrm{h}=$ width of each winding $=0.2 \mathrm{~cm}$ $\mathrm{b}=$ height of the windings $=0.8 \mathrm{~cm}$

$\mathrm{S}_{\mathrm{c}}=$ area of the center leg $=0.542 \mathrm{~cm}^{2}$

$\mathrm{S}_{\mathrm{o}}=$ area of the outer legs $=1.04 \mathrm{~cm}^{2}$

$\ell_{g}=$ length of the spacer gap $=0.28 \mathrm{~mm}$

The predicted electric circuit model (physical model) obtained by substitution of these values into Eqs. (39), (40), and (41) is shown in Fig. 19.

To experimentally verify the model, the four parameters $\mathrm{L}_{\mathrm{c}}, \mathrm{L}_{\mathrm{o}}, l$, and $\mathrm{N}$ can be derived from a set of impedance and voltage ratio measurements [7]. The electric circuit model derived from these measurements is shown in Fig. 19 , and compares favorably with the predicted results.

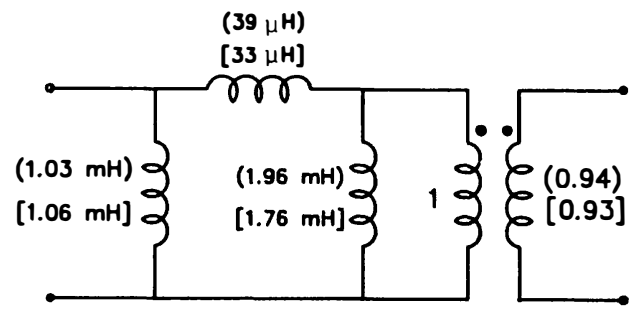

Fig. 19. Predicted () vs. measured [] electric circuit model values for two-winding side by side arrangement on a P-2213 pot core with a $0.28 \mathrm{~mm}$ spacer gap.

The second example is a similar pot core ( $P-2213)$ but with a very large center leg gap only. The length of the gap is $1.2 \mathrm{~mm}$, which for a core this size $(\approx 22 \mathrm{~mm} \times 13 \mathrm{~mm})$ is significantly large. Since only a center leg gap is used, the associated inductance of the outer legs $\mathrm{L}_{\circ}$ in Fig. 18 is very large with respect to $\mathrm{L}_{c}$ and $\mathrm{can}$ be neglected.

The predicted and measured electric circuit model values are illustrated in Fig. 20, where $\mathrm{L}_{\mathrm{o}} \approx \infty$. Although the errors between the measured and the predicted values have

increased, (probably because of the large size of the center leg gap), the measurements still compare well with the predictions.

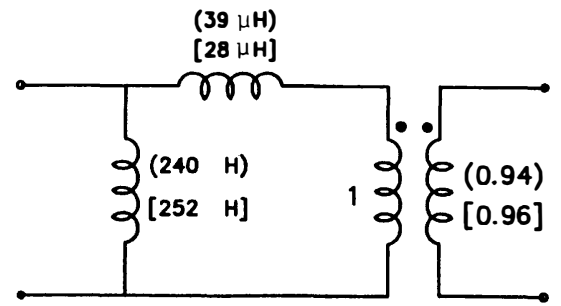

Fig. 20. Predicted () vs. measured [] electric circuit model values for two-winding side by side arrangement on a P-2213 pot core with a $1.2 \mathrm{~mm}$ center $\mathrm{leg}$ gap. 
Another commonly used two-winding configuration is the top-bottom arrangement illustrated in Fig. 21. The physical model of this structure is obtained the same way as before. The flux pattern inside the core and the windings is derived using Ampere's law and the integration path shown in Fig. 21. The field intensity and the flux pattern throughout the core and the windings are also illustrated in the figure.
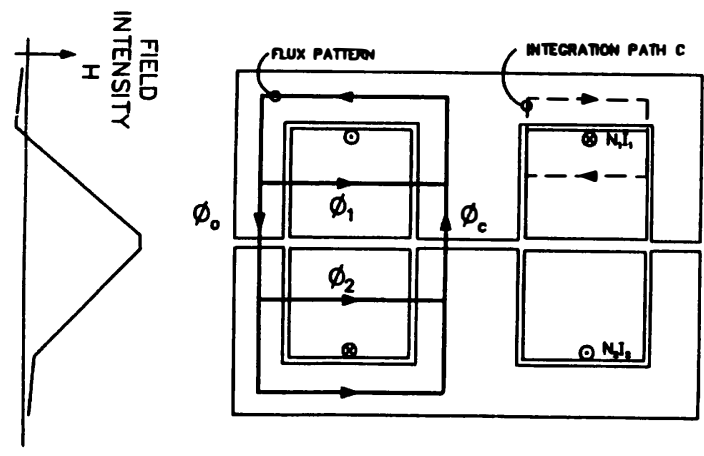

Fig. 21. Illustration of integration path, field intensity, and flux pattern inside two-winding top-bottom arrangement.

The infinitely small sources $\mathrm{N}_{1} \mathrm{I}_{1}$ and $\mathrm{N}_{2} \mathrm{I}_{2}$ are positioned at the extremes of the windings as indicated in Fig. 21. The fluxes enclosed by each one of these mmf sources (flux equations) are obtained from the flux pattern in Fig. 21 and are given by

$$
\begin{aligned}
& \phi_{\mathrm{N}_{1}}=\phi_{\mathrm{c}}+\phi_{1} \\
& \phi_{\mathrm{N} 2}=\phi_{0}-\phi_{2}
\end{aligned}
$$

Also, from the figure,

$$
\phi_{\mathrm{c}}=\phi_{\mathrm{o}}
$$

Equations (43) through (45) are used to construct the reluctance circuit model shown in Fig. 22. The electric circuit model derived from the reluctance model is illustrated in Fig. 23.

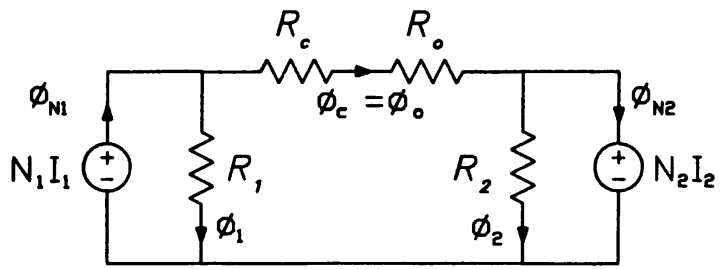

Fig. 22. Reluctance circuit model for twowinding top-bottom arrangement.

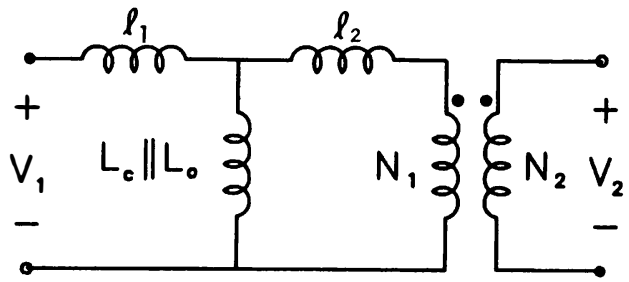

Fig. 23. Electric circuit model for twowinding top-bottom arrangement.

Because of the series connection of $R_{\mathrm{c}}$ and $R_{\circ}$ in the reluctance circuit, the corresponding inductances $\mathrm{L}_{c}$ and $\mathrm{L}_{o}$ in the electric circuit model appear as a parallel combination.

The expressions for $L_{c}$ and $L_{o}$ are again given by Eqs. (39) and (40) since they also represent the inductances of the center and the outer leg gaps.

The leakage inductances $\ell_{1}$ and $\ell_{2}$ can be estimated by shorting the secondary and calculating the amount of energy stored in each one of the windings. Evaluation of Eq. (4) throughout the volume of each one of the windings give

and

$$
\ell_{1}=\frac{\mu_{0}}{3} \mathrm{~N}_{1}^{2} \frac{\mathrm{b}_{1}}{\mathrm{~h}} \mathrm{MLT}
$$

$$
\ell_{2}=\frac{\mu_{\mathrm{o}}}{3} \mathrm{~N}_{1}^{2} \frac{\mathrm{b}_{2}}{\mathrm{~h}} \mathrm{MLT}
$$

where MLT $=2 \pi\left(r_{0}+h / 2\right)$ is the mean length per turn of the bobbin.

\subsection{Examples}

A small pot core (P-2213) with a center leg gap was used for this example. The windings are wound on a split bobbin as shown in Fig. 21.

The predicted and measured electric circuit models are shown in Fig. 24. The measurements compare favorably with the predictions.

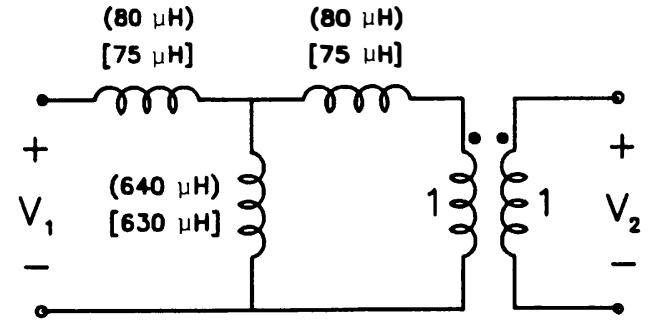

Fig. 24. Predicted () vs. measured [] electric circuit model values for two-winding top-bottom arrangement on a P-2213 pot core with a $0.45 \mathrm{~mm}$ center leg gap. 
There are several three and four-winding extensions of the side by side and the topbottom arrangements [7]. Only two will be discussed in this paper.

\subsection{Three-Winding Side by side Arrangement}

The first example is a three-winding extension of the side by side arrangement discussed in Section 5. It is illustrated in Fig. 25.

The flux pattern inside the structure is determined as before, and shown in Fig. 25. The flux equations are given by

$$
\begin{aligned}
& \phi_{\mathrm{N} 1}=\phi_{\mathrm{c}} \\
& \phi_{\mathrm{N} 2}=\phi_{\mathrm{c}}-\left(\phi_{1}+\phi_{2}\right) \\
& \phi_{\mathrm{N} 3}=\phi_{\mathrm{c}}-\left(\phi_{1}+\phi_{2}+\phi_{3}\right) \\
& \phi_{\mathrm{c}}=\phi_{\mathrm{o}}+\phi_{1}+\phi_{2}+\phi_{3}
\end{aligned}
$$

Substitution of Eq. (51) into Eqs. (48) through (50) gives the additional equations

$$
\begin{aligned}
& \phi_{\mathrm{N} 1}=\phi_{\circ}+\phi_{1}+\phi_{2}+\phi_{3} \\
& \phi_{\mathrm{N} 2}=\phi_{\circ}-\phi_{3} \\
& \phi_{\mathrm{N} 3}=\phi_{0}
\end{aligned}
$$

Equations (48) through (54) are used to construct the reluctance model illustrated in Fig. 26. The corresponding electric circuit model is shown in Fig. 27.

The inductances $\mathrm{L}_{c}$ and $\mathrm{L}_{o}$ in the electric circuit model correspond to the center and outer legs gaps respectively, and are given by Eqs. (39) and (40).

The leakage element $\ell_{12}$ is the leakage inductance between the primary $\mathrm{N}_{1}$ and the secondary $\mathrm{N}_{2}$, which is basically the same as the leakage inductance of the two-winding side by side arrangement given in Eq. (41).

The leakage inductance $\ell_{23}$ in Fig. 27 represents the energy storage capabilities between $\mathrm{N}_{2}$ and $\mathrm{N}_{3}$. It can be computed by opening the primary $N_{1}$, shorting the secondary $N_{2}$, and exciting $\mathrm{N}_{3}$ with a current source [7].

If $h_{1}=h_{2}=h_{3}=h / 3$, and $\delta_{1}, \delta_{2} \ll<$, the expressions for $\ell_{12}$ and $\ell_{23}$ reduce to

$$
\begin{aligned}
& \ell_{12}=\frac{4}{9} \pi \mu_{0} \mathrm{~N}_{1}^{2} \frac{\mathrm{h}}{\mathrm{b}}\left(\mathrm{r}_{\mathrm{o}}+\mathrm{h} / 3\right) \\
& \ell_{23}=\frac{4}{9} \pi \mu_{0} \mathrm{~N}_{3}^{2} \frac{\mathrm{h}}{\mathrm{b}}\left(\mathrm{r}_{0}+2 \mathrm{~h} / 3\right)
\end{aligned}
$$

Finally, it may be noted from the electric circuit model in Fig. 27 that there is no leakage inductance element in series with $\mathrm{N}_{2}$, which is because, physically, $\mathrm{N}_{2}$ is "sandwiched" between $\mathrm{N}_{1}$ and $\mathrm{N}_{3}$, as illustrated in Fig. 25. Therefore any flux enclosed by $\mathrm{N}_{2}$ is also approximately enclosed by either $\mathrm{N}_{1}$ or $\mathrm{N}_{3}$. However, the energy storage capability of $\mathrm{N}_{2}$ is represented in the electric circuit model by $\ell_{12}$ and $\ell_{23}$. For example, if $\mathrm{N}_{3}$ is left open, $\ell_{12}$ simply becomes the leakage inductance between $\mathrm{N}_{1}$ and $\mathrm{N}_{2}$. If $\mathrm{N}_{1}$ is open, $\ell_{23}$ is the leakage inductance between $\mathrm{N}_{2}$ and $\mathrm{N}_{3}$.

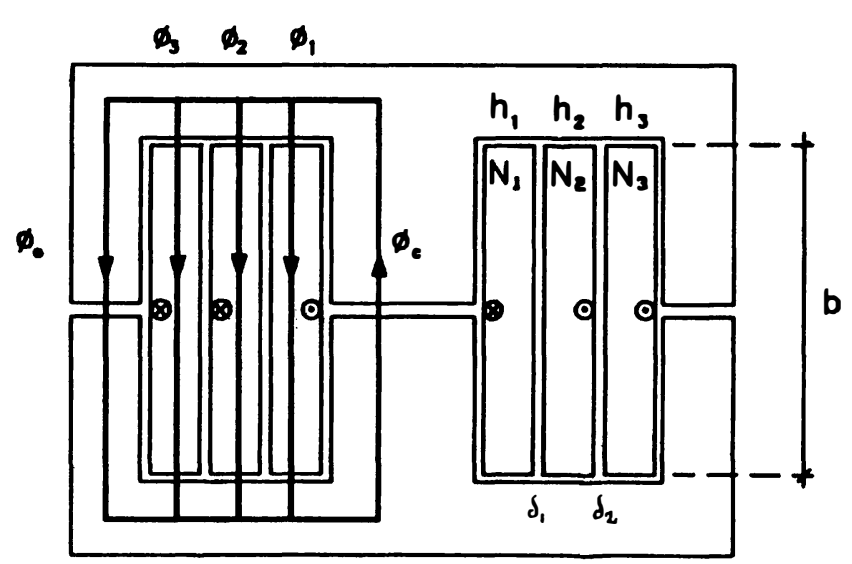

Fig. 25. Flux pattern inside three-winding side by side arrangement.

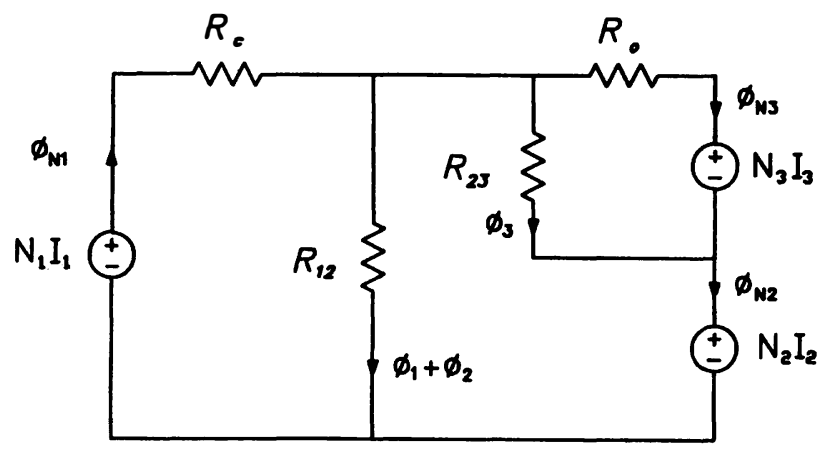

Fig. 26. Reluctance model for three-winding side by side arrangement.

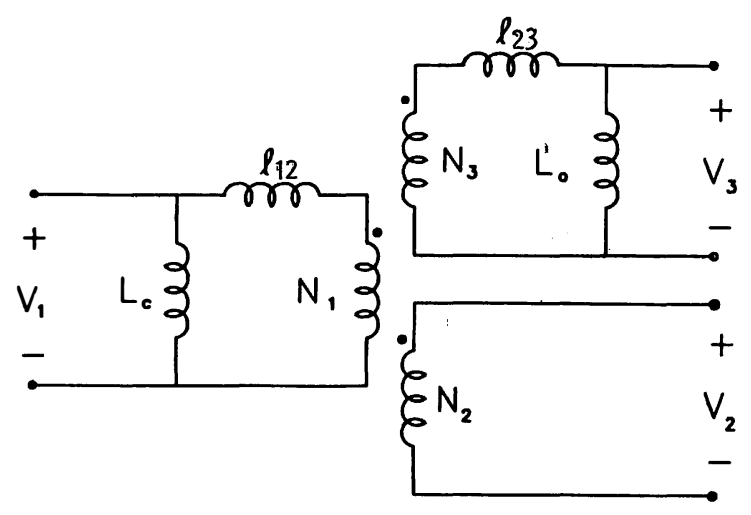

Fig. 27. Electric circuit model for threewinding side by side arrangement. 


\subsection{Four-Winding Top-Bottom Arrangement}

A four-winding extension of the top-bottom arrangement discussed in Section 6 is

illustrated in Fig. 28(a). The electric circuit model is derived in the same way as all the previous cases and is illustrated in Fig. 28(b).

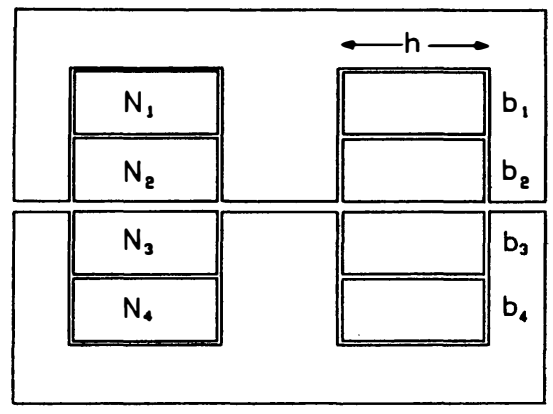

(a)

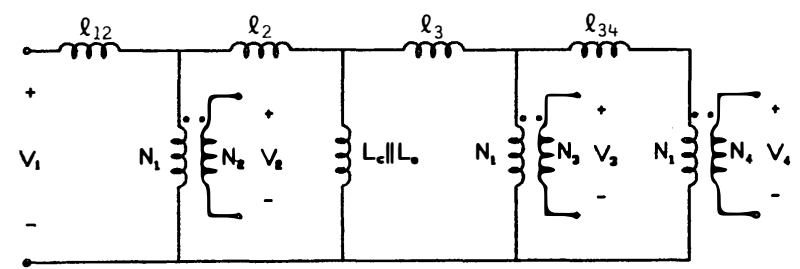

(b)

Fig. 28. Four winding extension of the topbottom arrangement (a) and the corresponding electric circuit model (b).

The expression for the leakage parameters in the model are given in [7].

\subsection{Example}

A large P-4229 ferrite pot core with a 1.4 $\mathrm{mm}$ spacer gap was wound as shown in Fig. 25. The three windings are of the same width, and the separations between them are negligible. The predicted and measured electric circuit model values are shown in in Fig. 29.

Other three and four-winding examples were also verified, and the measurements always compared favorably with the predictions.

\subsection{Other Extensions of Multiple-Winding Arrangements}

Further extensions of the arrangements previously discussed are possible, and are in general treated the same way as all the previous cases. The general results are similar, and further investigations of these give no additional qualitative information.

Other configurations with different types of cores (toroids etc.) and different winding arrangements (sectionalized, foils, etc) were also investigated. The results are discussed in [7].
$(32 \mu \mathrm{H})$ [31 $\mu \mathrm{H}$ ]

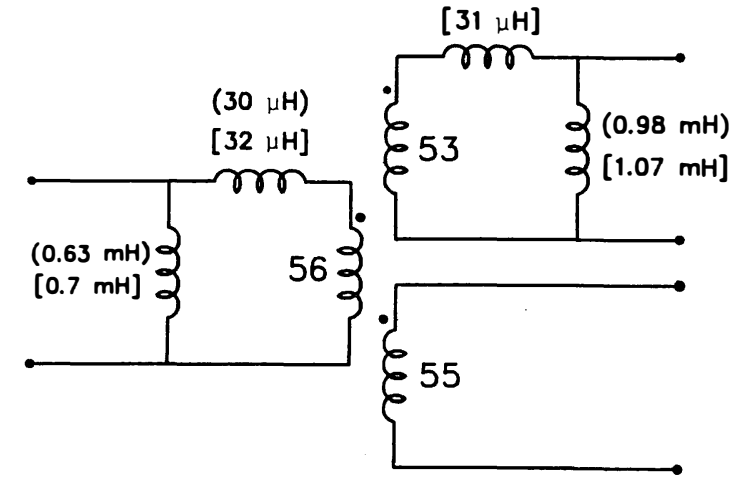

Fig. 29. Predicted () vs. measured [] electric circuit model values for threewinding side by side arrangement on a P-4229 pot core with a $1.4 \mathrm{~mm}$ spacer gap.

$8 \quad$ Conclusions

A new modelling method for magnetic circuits is presented in this paper. The electric circuit models (physical models) obtained with this method incorporate adequate information about the correct distribution of leakage energy in the windings of a magnetic structure, the presence of gaps throughout the magnetic path of the core, and the type of cores used.

The electric circuit models obtained with this method are physically natural, i.e. there is a one to one correspondence between the elements in the model and corresponding physical parameters in the original magnetic structure.

Several commonly used arrangements such as toroids with uniformly distributed turns, and the conventional side by side and top-bottom bobbin core arrangements with multiple windings were modelled with the new technique. The measured electric circuit model values always compared favorably with the predicted physical values derived with the new method.

Physical models for magnetic circuits can be used in a number of switching applications to enhance and facilitate what is usually a very lengthy and inefficient design process. This is specially the case when second-order specifications such as magnetic leakage are involved in the design process. Some of these applications are minimization of magnetic leakage effects in isolation transformers, and minimization of current ripple in coupled inductors. 
[1] Slobodan Ćuk, "Switching Dc-to-Dc Converter with Zero Input or Output Current Ripple," IEEE Industry Applications Society Annual Meeting, 1978 Record, pp 1131-1146.

[2] Slobodan Ćuk, "A New Zero-Ripple Switching DC-to-Dc Converter and Integrated Magnetics," IEEE Power Electronics Specialists Conference, 1980 Record, pp. $12-32$.

[3] Slobodan Ćuk and William M. Polivka, "Analysis of Integrated Magnetics to Eliminate Current Ripple in Switching Converters," Proceedings of the Sixth International PCI' 83 Conference, April 1983.

[4] R. D. Middlebrook and Slobodan Ćuk, "Isolation and Multiple Output Extensions of a New Optimum Topology Switching Dc-toDc Converter," IEEE Power Electronics Specialists Conference, 1978 Record, pp. 256-264.

[5] Shi-Ping Hsu, "Problems in Analysis and Design of Switching Regulators," Ph.D. Thesis, California Institute of Technology, Department of Electrical Engineering, Pasadena, California, September 13, 1979.

[6] William M. Polivka, "Applications of Magnetics to Problems in Switched-Mode Power Converters," Ph.D. Thesis, California Institute of Technology, Department of Electrical Engineering, Pasadena, California, February 7, 1984.

[7] Abraham A. Dauhajre, "Modelling and Estimation of Leakage Phenomena in Magnetic Circuits," Ph.D. Thesis, California Institute of Technology, Pasadena, California, April 22, 1986. 